\title{
Correction
}

\section{Correction: Voting-Based Cancer Module Identification by Combining Topological and Data-Driven Properties}

The PLOS ONE Staff

There is an error in the Correction published on January 30, 2014. The correction reads that "Figures S7 and S8 were interchanged." This is correct, however, Figure S8 is also incorrect and the correct file can be downloaded below.

\section{Supporting Information}

Figure S8. Distributions of topological and data-driven properties in merging pre-modules. (A) and (G) are distributions of topological property values of all pairs of premodules, and (B) and (D) are distributions of data-driven property values of all pairs of pre-modules, for GBM and OVC, respectively.

(TIF)

\section{Reference}

1. Azad AKM, Lee H (2013) Voting-Based Cancer Module Identification by Combining Topological and Data-Driven Properties. PLoS ONE 8(8): e70498. doi:10.1371/journal.pone.0070498

2. Azad AKM, Lee H (2014) Correction: Voting-Based Cancer Module Identification by Combining Topological and Data-Driven Properties. PLoS ONE 9(1): 10.1371/annotation/9a79fb7e-dce5-4525-9df5-dc130679c155.

Citation: The PLOS ONE Staff (2014) Correction: Voting-Based Cancer Module Identification by Combining Topological and Data-Driven Properties. PLoS ONE 9(4): e96883. doi:10.1371/journal.pone.0096883

Published April 28, 2014

Copyright: () 2014 The PLOS ONE Staff. This is an open-access article distributed under the terms of the Creative Commons Attribution License, which permits unrestricted use, distribution, and reproduction in any medium, provided the original author and source are credited. 